\title{
The relationship of mood status, quality of life, and dietary intake with migraine symptoms among women with migraine
}

\author{
Fatemeh Moradi $^{\circledR}{ }^{\circledR}$ Siavash Fazelian $^{2}{ }^{\circledR}$, Fariborz Khorvash $^{3}{ }^{\circledR}$, Gholamreza Askari $^{(\circledR)}$ \\ ${ }^{1} \mathrm{MSc}$ Student, Department of Community Nutrition, School of Nutrition and Food Science, Isfahan University of Medical \\ Sciences, Isfahan, Iran \\ ${ }^{2}$ Clinical Research Development Unit, Ayatollah Kashani Hospital, Shahrekord University of Medical Sciences, Shahrekord, Iran \\ ${ }^{3}$ School of Medicine, Isfahan University of Medical Sciences, Isfahan, Iran \\ ${ }^{4}$ Food Security Research Center and Department of Community Nutrition, School of Nutrition and Food Science, Isfahan \\ University of Medical Sciences, Isfahan, Iran
}

\begin{abstract}
Background and aims: Migraine is a neurologic disorder with wide global spread. Quality of life (QOL) and dietary factors are important parameters in migraine management. The aim of this study was to evaluate the relationship of mood status, QOL, and dietary intake with migraine symptoms among women with migraine. Methods: This cross-sectional study was conducted on 143 women with migraine aged 20-40 years who were randomly selected from two clinics in Isfahan, Iran. Data were collected using the Food Frequency Questionnaire for Assessing Dietary Patterns, a visual analogue scale for migraine headaches, the MigraineSpecific Quality of Life Questionnaire, and the Depression Anxiety Stress Scale. The serum level of calcitonin gene-related peptide (CGRP) was also measured.

Results: Participants' age and number of sleeping hours per 24 hours had significant relationship with migraine severity, depression and anxiety had significant relationship with migraine severity and the duration of migraine attacks, and QOL had significant relationship with migraine severity and the duration and frequency of migraine attacks. Daily intake of riboflavin also had significant relationship with frequency of migraine attacks, while daily intake of water had significant relationship with migraine severity $(P<0.05)$. However, serum level of CGRP had no significant relationship with migraine $(P>0.05)$. The relationships of vitamin $\mathrm{D}$ and magnesium intake with depression were also significant $(P<0.05)$.

Conclusion: Serum level of CGRP has no significant relationship with migraine attacks, while depression, anxiety, QOL, and magnesium and vitamin D intake have significant relationship with migraine attacks.

Keywords: Migraine, Depression, Mood status, Quality of life, Dietary intake
\end{abstract}

\author{
*Corresponding Author: \\ Gholamreza Askari, \\ Department of Community \\ Nutrition, School of Nutrition \\ and Food Science, Isfahan \\ University of Medical Scienc- \\ es, Isfahan, Iran. \\ Tel: (+98) 3137923171 \\ Email: askari@mui.ac.ir
}

Received: 14 December 2020 Accepted: 12 July 2021 ePublished: 30 September 2021

\section{Introduction}

Migraine is a highly prevalent neurologic disorder and the seventh leading cause of disability in the world (1). Almost $14 \%$ of people around the world experience migraine headaches (2). The average prevalence of migraine in Iran is also 14\% (3). Migraine headaches are usually unilateral and throbbing and are associated with fatigue, restlessness, concentration impairment, disability (4), and mood disorders such as depression and anxiety which increase the risk of disability, activity limitation, and quality of life (QOL) impairment (5). In one third of patients, migraine headaches are preceded by aura which is a focal neurological phenomenon characterized by numbness in the face and the hands, unilateral muscular tremor and weakness, and difficulty in speaking (6). The high prevalence of migraine and its significant effects on the different aspects of life highlight the importance of the timely use of safe and effective treatments. The most basic goal of migraine treatment is to improve QOL (7).

The most important factors contributing to migraine include genetic factors, sleep disorders, anxiety, stress, hypertension, disturbances of the monoaminergic neurotransmitters (including serotonin and dopamine), and mitochondrial disorders. Moreover, recent studies provided evidence regarding the relationship of calcitonin gene-related peptide (CGRP), neurological inflammation, and improper nutrition with migraine and recommended further studies to produce firmer evidence in this area $(8,9)$. CGRP is a 37 -aminoacid neuropeptide with wide spread in the central and peripheral nervous systems. Its secretion increases during migraine attacks and is associated with better pain transmission, higher sensitivity of pain pathways, and neurogenic inflammation (10).

Improper nutrition is a key factor in the onset of migraine attacks. Therefore, proper nutrition can facilitate effective migraine management. The American Neurological Association introduced riboflavin, magnesium, and coenzyme Q10 as supplements with potential positive effects on migraine prevention, while some studies reported that these supplements had no significant relationship with migraine $(11,12)$.

Although evidence recommends the high prevalence of

(C) 2021 The Author(s); Published by Shahrekord University of Medical Sciences. This is an open-access article distributed under the terms of the Creative Commons Attribution License (http://creativecommons.org/licenses/by/4.0), which permits unrestricted use, distribution, and reproduction in any medium, provided the original work is properly cited. 
psychological problems among patients with migraine, no study had yet evaluated the relationship of migraine with QOL measured using a migraine specific questionnaire. Therefore, the present study was conducted to bridge this gap. The aim of the study was to evaluate the relationship of mood status, QOL, and dietary intake with migraine symptoms among women with migraine.

\section{Methods \\ Design}

This cross-sectional study was conducted in 2019-2020 on 143 women with migraine randomly selected from Noor and Imam Musa Sadr clinics, Isfahan, Iran. Inclusion criteria were a definite diagnosis of migraine established by a neurologist based on the criteria of the International Classification of Headache Disorders (13), an age of 2040 years, no pregnancy or breastfeeding, no affliction by serious physical or mental disorders, and agreement for participation.

Sample size was calculated with a depression prevalence of $25 \%$, a confidence level of $95 \%$, a power of $80 \%$, and a $d$ of $10 \%(14,15)$. Sample size calculation formula (Eq. 1) revealed that with these parameters, 143 participants were needed for the study.

$$
n=\frac{\left(Z_{1-\alpha / 2}+Z_{1-\beta}\right)^{2} P(1-P)}{d^{2}}
$$

\section{Data collection instruments}

Study data were collected using the Food Frequency Questionnaire for Assessing Dietary Patterns, a visual analogue scale, the Migraine-Specific Quality of Life Questionnaire, and the Depression Anxiety Stress Scale. The Food Frequency Questionnaire for Assessing Dietary Patterns is a valid questionnaire for the assessment of dietary patterns and validation of other questionnaires on diet (16). In the present study, nutrition specialists trained participants how to complete this questionnaire and asked them to use graduated measures and pots for the accurate measurement of consumed food stuff. Participants completed this questionnaire on a daily basis and then, the amount of their consumed food stuff in one holiday and two non-holiday days was averaged. Values documented in the questionnaires were changed into grams and then, gram values were entered into the Nutritionist IV software to analyze and calculate consumed nutrients. Finally, the amounts of received calorie, macronutrients (i.e. carbohydrate, protein, and fat), and micronutrients (i.e., vitamin $\mathrm{D}$, riboflavin, magnesium, and water) were extracted and reported.

A visual analogue scale (numbered 1-10) was used for assessing migraine severity. The scale also included items on the frequency of migraine attacks per month, length of each attack, and type of migraine (with or without aura). A neurologist completed this scale for participants.

The Migraine-Specific Quality of Life Questionnaire was developed by Glaxo Wellcome to assess the effects of migraine on QOL (17). It has fourteen items on QOL in the past one month. Items are scored from 1 ("Never") to 6 ("Always"). The possible total score of this questionnaire is $14-84$, with higher scores showing better QOL. Masjedi et al. assessed and confirmed the validity and reliability of the Persian version of this questionnaire and reported that its Cronbach's alpha values among patients with all types of migraine, patients with chronic migraine, and patients with episodic migraine were $0.92,0.91$, and 0.92 , respectively (17).

The Depression Anxiety Stress Scale was used for mood status assessment. As a valid instrument to assess the symptoms of negative emotions, this scale has 21 items in three seven-item subscales. Items are scored from zero ("Does not apply to me at all") to 3 ("Applies to me very much"). As this scale is the short form of its 42 -item version, its scores should be doubled for interpretation (18). The validity and reliability of this scale were confirmed in previous studies (19). A study in Iran also confirmed the validity and reliability of the Persian version of this scale and reported that the Cronbach's alpha values of the whole scale and its depression, anxiety, and stress subscales were $0.91,0.87,0.85$, and 0.89 , respectively.

\section{Blood sampling and laboratory tests}

A blood sample was obtained from each participant after a twelve-hour fasting period and was kept at a temperature of $-70^{\circ} \mathrm{C}$. Serum level of CGRP was measured through enzyme-linked immunosorbent assay (ELISA). The microplate of the ELISA kit was pre-coated with a CGRP-specific monoclonal antibody bound with biotin and avidin conjugated to horseradish peroxidase. Blood samples were added to the plate and incubated and then, TMB (3,3, 5,5;-tetramethylbenzidine) substrate was added. Accordingly, plates with CGRP, biotin antibody, and Avidin showed color change. Enzymesubstrate reaction was ended by adding sulfuric acid and spectrophotometric measurement at a wave length of $450 \pm 2$ nanometer and then, the concentration of CGRP in samples was compared with the standard curve of optic density variations.

\section{Data analysis}

The SPSS software (v. 20.0) was used for data analysis. Data were described using mean and standard deviation. The one-way analysis of variance was used to compare patients with aura and patients without aura respecting the baseline values of the study variables. Moreover, linear regression analysis was conducted to assess relationships among the study variables adjusted for the effects of potential confounders. The level of significance was set at less than 0.05 .

\section{Results}

The mean of participants' age was $35.87 \pm 7.0$ years. The mean scores of their depression, anxiety, and stress were respectively $17.01 \pm 10.14,15.46 \pm 8.68$, and $23.16 \pm 10.16$, 
denoting moderate depression, anxiety, and stress. Group comparisons revealed no significant differences between patients with and without aura respecting their demographic characteristics, mood status, QOL, migraine type, and migraine severity $(P>0.05$; Table 1$)$.

Linear regression analysis revealed that after adjusting the effects of potential confounders, participants' age and number of sleeping hours per 24 hours had significant inverse relationship with migraine severity, the mean scores of depression, anxiety, and stress had significant relationship with migraine severity and the duration of migraine attacks, and the mean score of QOL had significant relationship with migraine severity and the frequency and duration of migraine attacks $(P<0.05)$. However, the serum level of CGRP had no significant relationship with migraine severity and the frequency and duration of migraine attacks $(P>0.5$; Table 2$)$.

The results of linear regression analysis also showed that after adjusting the effects of potential confounders, the serum levels of vitamin D and magnesium had significant relationship with depression, daily intake of riboflavin had significant inverse relationship with the frequency of migraine attacks, and daily intake of water had significant inverse relationship with migraine severity $(P<0.05$; Table 3).

\section{Discussion}

This study evaluated the relationship of mood status, QOL, and dietary intake with migraine symptoms among women with migraine. Findings showed that the number of sleeping hours per 24 hours had significant inverse relationship with migraine severity. Previous studies also reported that sleep quality among patients with migraine was lower than healthy individuals and had significant relationship with the frequency of migraine attacks, though its exact mechanism was reported to be unknown $(20,21)$. Sleep disorders can stimulate migraine attacks, can be aggravated by migraine attacks, or can have a same pathophysiological mechanism (22).

We also found that depression and anxiety had significant relationship with migraine severity and the frequency of migraine attacks. Previous studies also reported the high prevalence of depression and anxiety among patients with migraine $(23,24)$. Moreover, a study on 588 patients revealed that depression and anxiety had significant positive relationship with the frequency of migraine attacks

Table 1. Comparison of patients with aura and patients without aura respecting their characteristics

\begin{tabular}{lccc}
\hline \multirow{2}{*}{ Characteristics } & & Mean \pm Standard error & Total \\
\cline { 2 - 4 } & Migraine with aura & Migraine without aura & $35.87 \pm 7.0$ \\
\hline Age $(\mathrm{y})$ & $36.62 \pm 6.2$ & $35.53 \pm 7.34$ & $27.55 \pm 4.65$ \\
\hline Body mass index $\left(\mathrm{kg} / \mathrm{m}^{2}\right)$ & $28.02 \pm 4.84$ & $27.33 \pm 4.57$ & $7.31 \pm 1.99$ \\
\hline Sleeping hours per $24 \mathrm{~h}$ & $7.44 \pm 1.83$ & $129.77 \pm 179.59$ & $120.28 \pm 161.64$ \\
\hline Serum level of CGRP & $99.6 \pm 112.13$ & $6.88 \pm 1.55$ & $6.93 \pm 1.56$ \\
\hline Migraine severity & $7.02 \pm 1.6$ & $8.96 \pm 4.64$ & $8.61 \pm 4.26$ \\
\hline Frequency of migraine attacks & $7.84 \pm 3.2$ & $22.7 \pm 20.9$ & $20.57 \pm 19.23$ \\
\hline Duration of migraine attacks $(\mathrm{h})$ & $15.78 \pm 13.98$ & $16.47 \pm 10.09$ & $17.01 \pm 10.14$ \\
\hline Depression & $18.17 \pm 10.26$ & $14.88 \pm 8.37$ & $15.46 \pm 8.68$ \\
\hline Anxiety & $16.71 \pm 9.31$ & $22.53 \pm 10.07$ & 0.639 \\
\hline Stress & $24.53 \pm 10.33$ & $39.68 \pm 25.51$ & 0.096 \\
\hline Quality of life & $43.89 \pm 26.22$ & 0.358 \\
\hline
\end{tabular}

*The results of one-way analysis of variance.

Table 2. The results of linear regression analysis to assess the relationship of participants' characteristics with migraine characteristics

\begin{tabular}{|c|c|c|c|c|c|c|c|c|c|c|c|c|}
\hline \multirow[b]{2}{*}{ Characteristics } & \multicolumn{4}{|c|}{ Migraine severity } & \multicolumn{4}{|c|}{ Frequency of migraine attacks } & \multicolumn{4}{|c|}{ Duration of migraine attacks } \\
\hline & Beta & $\begin{array}{c}\text { Adjusted } \\
\text { beta* }\end{array}$ & $\begin{array}{c}95 \% \mathrm{Cl} \text { for } \\
\text { beta }\end{array}$ & $P$ value & Beta & $\begin{array}{l}\text { Adjusted } \\
\text { beta* }\end{array}$ & $\begin{array}{c}95 \% \mathrm{Cl} \text { for } \\
\text { beta }\end{array}$ & $P$ value & Beta & $\begin{array}{l}\text { Adjusted } \\
\text { beta* }\end{array}$ & $\begin{array}{c}95 \% \mathrm{Cl} \text { for } \\
\text { beta }\end{array}$ & $P$ value \\
\hline Age $(y)$ & -0.051 & -0.228 & $-0.98,-0.004$ & 0.035 & -0.048 & -0.079 & $-0.169,0.073$ & 0.436 & -0.407 & -0.148 & $-0.861,0.046$ & 0.078 \\
\hline $\begin{array}{l}\text { Body mass } \\
\text { index }\left(\mathrm{kg} / \mathrm{m}^{2}\right)\end{array}$ & 0.058 & 0.172 & $-0.003,0.118$ & 0.061 & 0.133 & 0.124 & $-0.043,0.269$ & 0.153 & -0.037 & -0.009 & $-0.618,0.545$ & 0.901 \\
\hline $\begin{array}{l}\text { Sleeping hours } \\
\text { per } 24 \mathrm{~h}\end{array}$ & -0.148 & -0.059 & $-0.283,-0.018$ & 0.042 & 0.031 & 0.014 & $-0.313,0.375$ & 0.859 & 0.185 & 0.019 & $-1.10,1.471$ & 0.776 \\
\hline $\begin{array}{l}\text { Serum level of } \\
\text { CGRP }\end{array}$ & 0.001 & 0.078 & $-0.001,0.002$ & 0.370 & 0.001 & 0.002 & $-0.003,0.006$ & 0.524 & -0.001 & -0.011 & $-0.017,0.015$ & 0.869 \\
\hline Depression & 0.210 & 0.191 & $0.008,0.467$ & 0.045 & 0.045 & 0.107 & $0.051,0.400$ & 0.022 & -0.064 & -0.034 & $-0.422,0.295$ & 0.726 \\
\hline Anxiety & 0.350 & 0.01 & $0.061,0.893$ & 0.002 & -0.071 & -0.145 & $-0.164,-0.021$ & 0.030 & 0.025 & 0.011 & $-0.321,0.370$ & 0.888 \\
\hline Stress & 0.000 & 0.001 & $-0.035,0.035$ & 0.995 & -0.094 & -0.224 & $-0.183,0.005$ & 0.059 & -0.490 & -0.259 & $-0.823,0.158$ & 0.06 \\
\hline Quality of life & -0.01 & -0.161 & $-0.12,-0.011$ & 0.023 & -0.061 & -0.366 & $-0.087,-0.034$ & 0.000 & -0.432 & -0.578 & $-0.531,-0.332$ & 0.000 \\
\hline
\end{tabular}

*Adjusted for the effects of potential confounders such as age and number of sleeping hours 


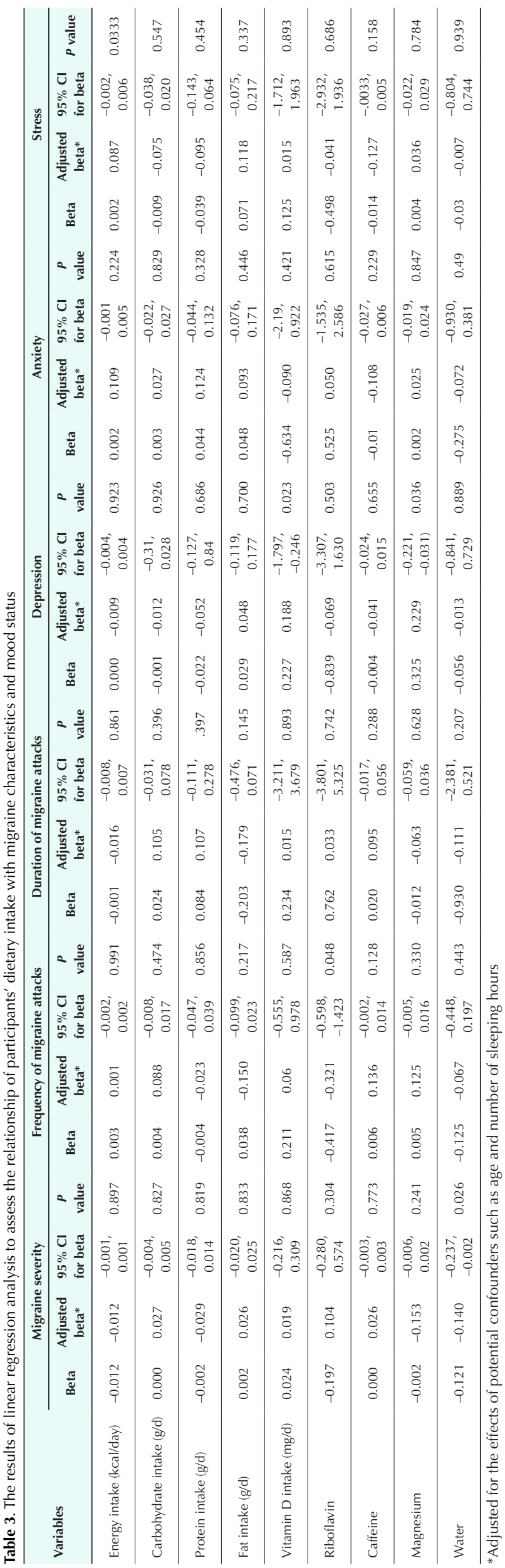

(25). Most migraine headaches are associated with mental and behavioral problems so that a bilateral relationship is considered between migraine and depression. Assessment of the symptoms appeared before and during migraine attacks in a study also revealed the probable involvement of hypothalamus (leading to depression, restlessness, and anxiety) and limbic system (leading to depression) (26). In line with our findings, a previous study showed that the number of days with migraine headaches per month had significant inverse relationship with QOL (27). Migraine is a severely debilitating disease and its significant effects on functional ability before, during, and after attacks significantly affect patients' QOL (28).

Study findings also revealed that riboflavin intake had significant relationship with the frequency of migraine attacks. Riboflavin catalyzes the activity of flavoenzymes in mitochondrial respiratory chain and thereby, alleviates clinical and biochemical anomalies in patients with mitochondrial metabolic errors. Given the abnormality of energy metabolism in the brain during migraine headaches, riboflavin is supposed to alleviate migraine headaches through this mechanism $(29,30)$. A metaanalysis also reported the effectiveness of riboflavin in significantly reducing the frequency and the duration of migraine attacks (31), though the first clinical study into the effects of riboflavin on migraine headaches among children reported no significant difference between the intervention and the placebo groups probably due to the small sample size of the study (32). Another study also reported that water intake reduced the duration of migraine attacks and hence, recommended that adequate water intake by patients with migraine can help alleviate migraine attacks (33).

Our findings also showed that magnesium intake had significant relationship with depression. Magnesium has significant roles in glutamatergic transmission in the limbic system and the cerebral cortex, inflammation and oxidative stress, and regulation of serotonin, dopamine, and noradrenalin, and thereby, can alleviate depression (33). In contradiction with our findings, a study reported that magnesium intake had no significant relationship with depression (34). This contradiction is attributable to the differences between these studies in terms of their participants' characteristics and the effects of potential confounders. Moreover, our findings indicated that vitamin D intake had significant relationship with depression. Different studies reported the effectiveness of vitamin $\mathrm{D}$ in alleviating the symptoms of depression $(34,35)$.

\section{Limitations and strengths}

This study had some limitations. For example, sample size was rather small due to financial and time limitations. The measurement of CGRP and micronutrients during migraine attacks was also not possible. Moreover, using a self-report questionnaire for assessing dietary patterns might have been associated with some measurement 
What does this paper contribute to the wider global clinical community?

- Effective management of depression and anxiety, consumption of a healthy and balanced diet, and intake of adequate water can improve QOL among patients with migraine.

- QOL improvement among patients with migraine can improve their functional ability and reduce the costs of migraine treatment.

biases. On the other hand, the use of the Migraine-Specific Quality of Life Questionnaire, assessment of factors with potential effects on migraine, and assessment of factors with potential effects on mood status were among the strengths of the present study.

\section{Conclusion}

This study suggests that serum level of CGRP has no significant relationship with migraine attacks, while depression, anxiety, QOL, and magnesium and vitamin D intake have significant relationship with migraine attacks.

\section{Conflict of Interests}

The authors declare no conflict of interests.

\section{Ethical approval}

The Ethics Committee of Isfahan University of Medical Sciences, Isfahan, Iran, approved this study (code: IR.MUI.RESEARCH. REC.1398.499). Participants were provided with adequate information about the study aim and were ensured of data confidentiality. Their questions were also answered and written informed consent was obtained from all of them. They were also informed that they could access the result of their dietary intake analysis and its interpretation at personal request at the end of the study.

\section{Acknowledgement}

We would like to thank all participants of the study as well as all individuals who collaborated with us during the study.

\section{References}

1. Henssen D, Derks B, van Doorn M, Verhoogt N, Van Cappellen van Walsum AM, Staats $P$, et al. Vagus nerve stimulation for primary headache disorders: an anatomical review to explain a clinical phenomenon. Cephalalgia. 2019;39(9):1180-94. doi: 10.1177/0333102419833076.

2. Stovner LJ, Nichols E, Steiner TJ, Abd-Allah F, Abdelalim A, Al-Raddadi RM, et al. Global, regional, and national burden of migraine and tension-type headache, 1990-2016: a systematic analysis for the Global Burden of Disease Study 2016. Lancet Neurol. 2018;17(11):954-76. doi: 10.1016/ s1474-4422(18)30322-3

3. Farhadi Z, Alidoost S, Behzadifar M, Mohammadibakhsh R, Khodadadi N, Sepehrian R, et al. The prevalence of migraine in Iran: a systematic review and meta-analysis. Iran Red Crescent Med J. 2016;18(10):e40061. doi: 10.5812/ircmj.40061.

4. Burstein R, Noseda R, Borsook D. Migraine: multiple processes, complex pathophysiology. J Neurosci. 2015;35(17):6619-29. doi: 10.1523/jneurosci.0373-15.2015.

5. Yalinay Dikmen P, Yavuz BG, Aydinlar El. The relationships between migraine, depression, anxiety, stress, and sleep disturbances. Acta Neurol Belg. 2015;115(2):117-22. doi: 10.1007/s13760-014-0312-0.
6. Cutrer FM, Olesen J. Migraines with aura and their subforms. In: Olesen J, Goadsby PJ, Ramadan NM, Tfelt-Hansen P, Welch KMA, eds. The Headaches. 3rd ed. Philadelphia, PA: Lippincott Williams \& Wilkins; 2006. p. 407-21.

7. Parohan $M$, Sarraf $P$, Javanbakht $M H$, Foroushani $A R$ Ranji-Burachaloo S, Djalali M. The synergistic effects of nano-curcumin and coenzyme Q10 supplementation in migraine prophylaxis: a randomized, placebo-controlled, double-blind trial. Nutr Neurosci. 2021;24(4):317-26. doi: 10.1080/1028415x.2019.1627770.

8. Martin VT, Fanning KM, Serrano D, Buse DC, Reed ML, Lipton RB. Asthma is a risk factor for new onset chronic migraine: results from the American migraine prevalence and prevention study. Headache. 2016;56(1):118-31. doi: 10.1111/head.12731.

9. May A, Schulte LH. Chronic migraine: risk factors, mechanisms and treatment. Nat Rev Neurol. 2016;12(8):455-64. doi: 10.1038/nrneurol.2016.93.

10. Lemos C, Alonso I, Barros J, Sequeiros J, PereiraMonteiro J, Mendonça D, et al. Assessing risk factors for migraine: differences in gender transmission. PLoS One. 2012;7(11):e50626. doi: 10.1371/journal.pone.0050626.

11. Chiu HY, Yeh TH, Huang YC, Chen PY. Effects of intravenous and oral magnesium on reducing migraine: a meta-analysis of randomized controlled trials. Pain Physician. 2016;19(1):E97112.

12. Orr SL. Diet and nutraceutical interventions for headache management: a review of the evidence. Cephalalgia. 2016;36(12):1112-33. doi: 10.1177/0333102415590239.

13. Taghdiri F, Togha M, Razeghi Jahromi S, Refaeian F. Cinnarizine for the prophylaxis of migraine associated vertigo: a retrospective study. Springerplus. 2014;3:231. doi: 10.1186/2193-1801-3-231.

14. Desouky DE, Zaid HA, Taha AA. Migraine, tension-type headache, and depression among Saudi female students in Taif University. J Egypt Public Health Assoc. 2019;94(1):7. doi: 10.1186/s42506-019-0008-7.

15. Lee SH, Kang Y, Cho SJ. Subjective cognitive decline in patients with migraine and its relationship with depression, anxiety, and sleep quality. J Headache Pain. 2017;18(1):77. doi: 10.1186/s10194-017-0779-1.

16. Rashidkhani B, Shahneshin M, Rafat R, Omidvar N, HoshiarRad A, Rezazadeh A, et al. Validity and reproducibility of a food frequency questionnaire for assessing dietary patterns in 18-45 years old women supported by health care in the north and east of Tehran. Iran J Endocrinol Metab. 2011;13(1):58-66. [Persian].

17. Zandifar A, Masjedi SS, Haghdoost F, Asgari F, Manouchehri N, Banihashemi M, Najafi MR, Ghorbani A, Zolfaghari B, Gholamrezaei A, Shaygannejad V. The psychometric properties of the persian migraine-specific quality of life questionnaire version 2.1 in episodic and chronic migraines. The Scientific World Journal. 2013.

18. Lovibond PF, Lovibond SH. The structure of negative emotional states: comparison of the Depression Anxiety Stress Scales (DASS) with the Beck Depression and Anxiety Inventories. Behav Res Ther. 1995;33(3):335-43. doi: 10.1016/00057967(94)00075-u.

19. Lovibond PF. Long-term stability of depression, anxiety, and stress syndromes. J Abnorm Psychol. 1998;107(3):520-6. doi: 10.1037//0021-843x.107.3.520.

20. Song TJ, Chu MK, Sohn JH, Ahn HY, Lee SH, Cho SJ. Effect of vitamin $\mathrm{D}$ deficiency on the frequency of headaches in migraine. J Clin Neurol. 2018;14(3):366-73. doi: 10.3988/ jcn.2018.14.3.366.

21. Sadeghniiat K, Rajabzadeh A, Ghajarzadeh M, Ghafarpour M. Sleep quality and depression among patients with migraine. Acta Med Iran. 2013;51(11):784-8. 
22. Dodick DW, Eross EJ, Parish JM, Silber M. Clinical, anatomical, and physiologic relationship between sleep and headache. Headache. 2003;43(3):282-92. doi: 10.1046/j.15264610.2003.03055.x.

23. Llop SM, Frandsen JE, Digre KB, Katz BJ, Crum AV, Zhang C, et al. Increased prevalence of depression and anxiety in patients with migraine and interictal photophobia. J Headache Pain. 2016;17:34. doi: 10.1186/s10194-016-0629-6.

24. Yalug I, Selekler M, Erdogan A, Kutlu A, Dundar G, Ankarali $\mathrm{H}$, et al. Correlations between alexithymia and pain severity, depression, and anxiety among patients with chronic and episodic migraine. Psychiatry Clin Neurosci. 2010;64(3):2318. doi: 10.1111/j.1440-1819.2010.02093.x.

25. Chu HT, Liang CS, Lee JT, Yeh TC, Lee MS, Sung YF, et al. Associations between depression/anxiety and headache frequency in migraineurs: a cross-sectional study. Headache. 2018;58(3):407-15. doi: 10.1111/head.13215.

26. Maniyar FH, Sprenger T, Monteith T, Schankin C, Goadsby PJ. Brain activations in the premonitory phase of nitroglycerintriggered migraine attacks. Brain. 2014;137(Pt 1):232-41. doi: 10.1093/brain/awt320.

27. Vo P, Fang J, Bilitou A, Laflamme AK, Gupta S. Patients' perspective on the burden of migraine in Europe: a crosssectional analysis of survey data in France, Germany, Italy, Spain, and the United Kingdom. J Headache Pain. 2018;19(1):82. doi: 10.1186/s10194-018-0907-6.

28. Steiner TJ, Gururaj G, Andrée C, Katsarava Z, Ayzenberg I, Yu SY, et al. Diagnosis, prevalence estimation and burden measurement in population surveys of headache: presenting the HARDSHIP questionnaire. J Headache Pain. 2014;15(1):3. doi: 10.1186/1129-2377-15-3.
29. Meissner K, Fässler M, Rücker G, Kleijnen J, Hróbjartsson A, Schneider A, et al. Differential effectiveness of placebo treatments: a systematic review of migraine prophylaxis. JAMA Intern Med. 2013;173(21):1941-51. doi: 10.1001/ jamainternmed.2013.10391.

30. Gasparini CF, Sutherland HG, Griffiths LR. Studies on the pathophysiology and genetic basis of migraine. Curr Genomics. 2013;14(5):300-15. doi: 10.2174/13892029113149990007.

31. Namazi N, Heshmati J, Tarighat-Esfanjani A. Supplementation with Riboflavin (vitamin B2) for migraine prophylaxis in adults and children: a review. Int J Vitam Nutr Res. 2015;85(1-2):7987. doi: 10.1024/0300-9831/a000225.

32. MacLennan SC, Wade FM, Forrest KM, Ratanayake PD, Fagan E, Antony J. High-dose riboflavin for migraine prophylaxis in children: a double-blind, randomized, placebocontrolled trial. J Child Neurol. 2008;23(11):1300-4. doi: 10.1177/0883073808318053.

33. Spigt $M$, Weerkamp $N$, Troost J, van Schayck CP, Knottnerus JA. A randomized trial on the effects of regular water intake in patients with recurrent headaches. Fam Pract. 2012;29(4):3705. doi: 10.1093/fampra/cmr112.

34. Menon V, Kar SK, Suthar N, Nebhinani N. Vitamin D and depression: a critical appraisal of the evidence and future directions. Indian J Psychol Med. 2020;42(1):11-21. doi: 10.4103/ijpsym.ijpsym_160_19.

35. Shaffer JA, Edmondson D, Wasson LT, Falzon L, Homma $\mathrm{K}$, Ezeokoli N, et al. Vitamin D supplementation for depressive symptoms: a systematic review and metaanalysis of randomized controlled trials. Psychosom Med. 2014;76(3):190-6. doi: 10.1097/psy.0000000000000044. 